(с) Самцов А.В. ${ }^{*}$, Соколовский Е.В. ${ }^{2}$, Теплюк Н.П. ${ }^{3}$, Белоусова И.Э. ${ }^{1}$, Кохан М.М. ${ }^{4}$, Матушевская Е.В. ${ }^{5}$

\author{
${ }^{1}$ Военно-медицинская академия им. С.М. Кирова \\ 194044, Россия, г. Санкт-Петербург, ул. Академика Лебедева, д. 6 \\ 2 Первый Санкт-Петербургский государственный медицинский университет им. акад. И.П. Павлова \\ 197022, Россия, г. Санкт-Петербург, ул. Льва Толстого, д. 6-8 \\ 3 Первый Московский государственный медицинский университет им. И.М. Сеченова \\ 119991, Россия, г. Москва, Трубецкая ул., д. 8, стр. 2 \\ 4 Уральский научно-исследовательский институт дерматовенерологии и иммунопатологии \\ 620076, Россия, г. Екатеринбург, ул. Щербакова, д. 8 \\ ${ }^{5}$ Академия постдипломного образования ФГБУ ФНКЦ ФМБА \\ 125371, Россия, г. Москва, Волоколамское шоссе, д. 91
}

Анализ различных классификаций пузырчатки показывает, что принципиальных различий между ними нет, основные отличия связаны с использованием различной терминологии в обозначении некоторых форм пузырчатки, наличием или отсутствием тех или иных подтипов в классификациях. Авторы предлагают использовать в клинической работе врачей дерматовенерологов, в учебных и научных целях, при разработке клинических рекомендаций следующую классификацию пузырчатки: 1) вульгарная пузырчатка (1.1. Вегетирующая пузырчатка); 2) листовидная пузырчатка (2.1. Эндемичная пузырчатка (Fogo selvagem), 2.2. Эритематозная пузырчатка (Senear - Usher)); 3) герпетифрормная пузырчатка; 4) паранеопластическая пузырчатка; 5) IgA-пузырчатка (5.1. Субкорнеальный пустулезный дерматоз, 5.2. Внутриэпидермальный нейтрофильный дерматоз).

Ключевые слова: пузырчатка, классификация, патофизиология.

Конфрликт интересов: авторы данной статьи подтвердили отсутствие конфрликта интересов, о котором необходимо сообщить.

Источник финансирования: работа выполнена и опубликована за счет финансирования по месту работы авторов.

Для цитирования: Самцов А.В., Соколовский Е.В., Теплюк Н.П., Белоусова И.Э., Кохан М.М., Матушевская Е.В. К вопросу о классификации пузырчатки. Вестник дерматологии и венерологии. 2021;97(2):09-15. doi: https://doi.org/10.25208/vdv1216 
$\mathrm{R}$ evisiting the question of pemphigus classification

\author{
(C) Alexey V. Samtsov ${ }^{1 *}$, Evgeny V. Sokolovskiy², Natalia P. Teplyuk ${ }^{3}$, Irena E. Belousova', Muza M. Kokhan4, \\ Elena V. Matushevskaya ${ }^{5}$
}

\author{
${ }^{1}$ S.M. Kirov Military Medical Academy \\ Akademika Lebedeva str., 6, 194044, Saint Petersburg, Russia \\ ${ }^{2}$ First Pavlov State Medical University of Saint Petersburg \\ Lev Tolstoy str., 6-8, 197022, Saint Petersburg, Russia \\ ${ }^{3}$ Sechenov University \\ Trubetskaya str., 8-2, 119991, Moscow, Russia \\ ${ }^{4}$ Ural Scientific Research Institute of Dermatovenerology and Immunopathology \\ Shcherbakova str., 8, 620076, Ekaterinburg, Russia \\ ${ }^{5}$ Academy of postgraduate education under FSBU FSCC of FMBA of Russia \\ Volokolamskoe hwy, 91, 125371, Moscow, Russia
}

Analysis of various classifications of pemphigus shows that there are no fundamental differences between them. The main distinctions consist in use of diverse terms in naming of some forms of pemphigus and in inclusion or exclusion of certain subtypes from the classifications. Authors propose to use the following classification in the dermatological clinical practice, for educational and scientific purposes and for clinical guidelines: 1) pemphigus vulgaris (1.1. Pemphigu s vegetans); 2) pemphigus foliaceus (2.1. Pemphigus endemic (Fogo selvagem), 2.2. Pemphigus erythematosus (Senear - Usher)); 3) herpetiform pemphigus; 4) paraneoplastic pemphigus; 5) IgA pemphigus (5.1. Subcorneal pustular dermatosis, 5.2. Intraepidermal neutrophilic dermatosis).

Keywords: pemphigus, classification, pathophysiology.

Conflict of interest: the authors of this article have confirmed that there is no conflict of interest to report.

Source of funding: the work was done and published through financing at the place of work of the authors.

For citation: Samtsov AV, Sokolovskiy EV, Teplyuk NP, Belousova IE, Kokhan MM, Matushevskaya EV. Revisiting the question of pemphigus classification. Vestnik Dermatologii i Venerologii. 2021;97(2):09-15.

doi: https://doi.org/10.25208/vdv1216 
Пемфригус (пузырчатка) представляет собой группу аутоиммунных буллезных дерматозов, характеризующихся вялыми пузырями на слизистых оболочках и/или коже, которые могут приводить к летальному исходу [1]. Патофизиология пузырчатки в настоящее время рассматривается как процесс образования патогенных аутоантител (обычно класca $\lg$, реже $\lg A$ ), направленных против различных белков десмосом кератиноцитов - десмоглеинов, десмоколлинов, плакинов, а соединение аутоантител с компонентами десмосом нарушает внутриэпидермальную адгезию, приводя к акантолизу и образованию пузырей на коже и/или слизистых оболочках, при этом активность заболевания тесно коррелирует с уровнем аутоантител в сыворотке крови [2, 3].

Современные представления о пемфигусе начали формироваться в 1953 г. с опубликования книги W. Lever "Pemphigus" [4]. Автор впервые диффреренцировал на основании клинико-гистологических критериев буллезный пемфигоид и вульгарный пемфигус. В дальнейшем W. Lever на основании гистологических данных выделил две фрормы пузырчатки в зависимости от уровня акантолиза - листовидную и ее вариант - себорейную, а также вульгарную и ее вариант - вегетирующую.

B 1975 г. S. Jablonska и соавт. впервые описали герпетиформную пузырчатку. Герпетиформная пузырчатка - редкий вариант пемфигуса, характеризующийся сочетанием клинической картины герпетиформного дерматита и иммунопатологических признаков листовидной или вульгарной пузырчатки [5].

В 1982 г. D. Wallach и соавт. выделили IgA пемфигус как самостоятельное заболевание [6]. IgA пемфигус представляет собой группу аутоиммунных интраэпидермальных буллезных дерматозов, характеризующихся везикулопустулезными высыпаниями, нейтрофильной инфильтрацией, акантолизом и циркулирующими аутоантителами против поверхности клеток эпидермиса [7]. В настоящее время выделяют два типа IgA пемфигуса: субкорнеальный пустулезный дерматоз (СПД) и интраэпидермальный нейтрофрильный дерматоз (ИНД) [7].

B 1990 г. G. Anhalt и соавт. впервые ввели термин «паранеопластическая пузырчатка» [8]. Паранеопластическая пузырчатка относится к группе аутоиммунных буллезных дерматозов и характеризуется поражением слизистых оболочек, полиморфными высыпаниями на коже и наличием опухоли внутренних органов [8, 9].

Все перечисленные выше дерматозы в настоящее время относятся к пузырчатке, несмотря на то что различаются по патогенезу и клинической картине. Это объясняет наличие разнообразных классификаций, наиболее известные из которых за последние 10 лет мы приводим ниже.

В 2010 г. в многотомном руководстве “Rook's Textbook of Dermatology" F. Wojnarowska $и$ V. A. Venning предложили следующую классификацию пузырчатки [10]:

\section{Вульгарная пузырчатка}

Вариант: вегетирующая пузырчатка

Листовидная пузырчатка

Вариант: герпетиформная пузырчатка

Вариант: эритематозная пузырчатка

Индуцированная пузырчатка

Внутриклеточный IgA-дерматоз

\section{Паранеопластическая пузырчатка}

В 2012 г. в монографии А. В. Самцова и И. Э. Белоусовой герпетиформная пузырчатка выделена как самостоятельная форма, а лекарственно-индуцированная пузырчатка отсутствует [11]:

1. Вульгарная пузырчатка

1.1. Вегетирующая пузырчатка (локализованная форма)

2. Листовидная пузырчатка

2.1. Эритематозная пузырчатка (локализованная форма)

2.2. Бразильская пузырчатка (Fogo selvagem) (эндемичная форма)

\section{3. Герпетиформная пузырчатка \\ 4. IgА-пузырчатка \\ 5. Паранеопластическая пузырчатка}

В 2015 г. в классификации, опубликованной в Европейском руководстве, выделили два типа IgA-пузырчатки [12]:

Пузырчатка вульгарная

Пузырчатка вегетирующая

Neumann-тип, характеризующийся периорифициальными папилломами

Hallopeau-тип, характеризующийся пустулами преимущественно в крупных складках

Пузырчатка листовидная

Пузырчатка эритематозная

Пузырчатка листовидная эндемичная

Пузырчатка паранеопластическая / паранеопластический аутоиммунный синдром

IgА-пузырчатка

Субкорнеальный пустулезный дерматоз

Интраэпидермальный нейтрофильный дерматоз

В 2016 г. была предложена наиболее развернутая классификация пузырчатки, в которой впервые выделили слизистый и слизисто-кожный типы вульгарной пузырчатки [13]:

Пузырчатка вульгарная

Слизистый тип

Слизисто-кожный тип

Пузырчатка вегетирующая

Neumann-тип

Hallopeau-тип

Пузырчатка листовидная

Пузырчатка эндемичная

Локализованная форма

Генерализованная форма

Пузырчатка эритематозная

Пузырчатка паранеопластическая / паранеопластический аутоиммунный синдром

IgA-пузырчатка

Субкорнеальный пустулезный дерматоз

Интраэпидермальный нейтрофильный дерматоз

Пузырчатка лекарственно-индуцированная

Пузырчатка паранеопластическая

В 2016 г. Н.В. Махнева, Н.П. Теплюк, Л.В. Белецкая в монографии «Аутоиммунная пузырчатка (от истоков развития до наших дней)» определили герпетиформную пузырчатку как вариант вульгарной пузырчатки [14]:

1. Вульгарная пузырчатка

1.1. Вегетирующая пузырчатка 
1.2. Герпетифрормная пузырчатка

\section{2. Листовидная пузырчатка}

2.1. Эндемичная Бразильская пузырчатка (Fogo selvagem)

2.2. Эритематозная пузырчатка (Senear - Usher)

3. Паранеопластическая пузырчатка

4. Лекарственно-индуцированная пузырчатка

5. IgА-зависимая пузырчатка

5.1. Субкорнеальный пустулез

5.2. Внутриэпидермальный нейтрофильный дерматоз

Члены рабочей группы сознательно не рассматривали и не проводили анализа фрорм пузырчатки, указанных в Международной классифрикации болезней (МКБ), так как последняя является не клинической, а международной статистической классификацией. В России МКБ принята как единый нормативный документ для учета заболеваемости, причин обращений населения в медицинские учреждения всех ведомств, причин смерти. МКБ является нормативным документом, обеспечивающим единство методических подходов и международную сопоставимость материалов - это опубликовано в преамбуле к действующей на территории России МКБ 10. Все это определяет ее значение и область использования. К сожалению, МКБ не отражает всего многообразия и возможных соотношений клинических ситуаций и диагнозов у пациента, именно поэтому во всем мире используются и будут применяться в клинической практике клинические классификации болезней и состояний, а их статистический учет будет проводиться с учетом принципов МКБ. Для облегчения работы врачей-дерматовенерологов мы приводим в таблице коды МКБ 10 и МКБ 11 (будет введена в действие в ближайшие годы), по которым проводится учет различных форм пузырчатки.

Анализ классификаций пузырчатки свидетельствует о том, что принципиальных различий между ними нет. Вместе с тем обращает на себя внимание различ- ная терминология некоторых фрорм пузырчатки, наличие или отсутствие тех или иных подтипов в классификациях. Это послужило основанием для обсуждения данной проблемы с целью создания единой отечественной классификации пузырчатки.

У экспертов возникли следующие вопросы, на которые после обстоятельного обсуждения были даны ответы.

1. Следует ли рассматривать вегетирующую пузырчатку как ограниченный вариант вульгарной пузырчатки или как самостоятельную фрорму с учетом различной клинической картины?

В подавляющем большинстве классификаций вегетирующая пузырчатка рассматривается как вариант вульгарной пузырчатки, поскольку различия в клинической картине объясняются интертригинозной локализацией. К тому же в обоих случаях выявляются аутоантитела к десмоглеину-3.

2. Целесообразно ли выделять две формы вегетирующей пузырчатки?

Мы не классифицировали вегетирующую пузырчатку по следующим соображениям. Вегетирующая пузырчатка (пиодермия) Hallopeau, по мнению некоторых авторов, по клинике напоминает вегетирующую пузырчатку Neumann с той лишь разницей, что в данном случае первичный морфологический элемент кожной сыпи представлен пустулой, а не пузырем [15]. Вместе с тем следует отметить, что вегетирующая пузырчатка встречается исключительно редко, количество наблюдений, представленных для сравнения, незначительно. В связи с этим, на наш взгляд, оправдана точка зрения М. Megahed, который считает, что различия между этими типами представляют собой лишь исторический интерес. Тем более что сам F.H. Hallopeau изменил свою точку зрения, рассмотрев описанный им тип как пустулезный вариант вегетирующего пемфигуса Neumann [16].

\begin{tabular}{|c|c|c|}
\hline & MKБ 10 & МКБ 11 \\
\hline ПУЗЫРЧАТКА & L10.0 & EB40 \\
\hline 1. Вульгарная пузырчатка & L10.0 & EB40.0 \\
\hline 1.1. Вегетирующая пузырчатка (локализованная форма) & L10.1 & EB40.0 \\
\hline \multirow{3}{*}{$\begin{array}{l}\text { 2. Листовидная пузырчатка } \\
\text { 2.1. Эндемичная пузырчатка (Fogo selvagem) } \\
\text { 2.2. Эритематозная пузырчатка (Senear — Usher) }\end{array}$} & L10.2 & EB40.1 \\
\hline & L10.3 & EB40.1 \\
\hline & L10.4 & EB40.1 \\
\hline 4. Паранеопластическая пузырчатка & L10.8 & EB40.2 \\
\hline $\begin{array}{c}\text { 5. IgA-пузырчатка } \\
\text { 5.1. Субкорнеальный пустулез } \\
\text { 5.2. Внутриэпидермальный нейтрофрильный дерматоз }\end{array}$ & L13.8 & EB40.Y \\
\hline
\end{tabular}


3. Рассматривать ли эритематозную (себорейную) пузырчатку как ограниченный вариант листовидной пузырчатки?

В подавляющем большинстве классификаций эритематозная (себорейная) пузырчатка относится к варианту листовидной пузырчатки. Важно также то, что в обоих случаях выявляются аутоантитела к десмоглеину-1.

4. Эндемичная пузырчатка - вариант листовидной или самостоятельная форма?

Большинство авторов рассматривает ее как эндемичный вариант листовидной пузырчатки в связи с тем, что аутоантигеном, как и при листовидной пузырчатке, является десмоглеин-1.

5. Рассматривать ли как самостоятельную форму лекарственно-индуцированную пузырчатку?

Вопрос о выделении индуцированной формы (индукторами могут быть не только лекарства, но и другие факторы, в частности солнечные лучи) дискутабельный. Мы считаем, что индуцированную форму выделять как отдельный вариант пузырчатки нецелесообразно по следующим причинам.

1. Индуцированная фрорма клинически и гистологически может проявляться как вульгарная, листовидная и герпетиформная пузырчатки.

2. Нет убедительного ответа на самый важный вопрос - связано ли разрешение дерматоза с отменой препарата.

3. Учитывая, что большинство пациентов заболевают в возрасте от 40 до 60 лет (высока вероятность полипрагмазии), четко определить конкретный препарат бывает сложно, а лечение проводить необходимо максимально рано по общим принципам терапии пузырчатки. Вместе с тем вполне оправдано при сборе анамнеза обращать особое внимание на возможную роль лекарственного препарата в развитии пузырчатки.

6. Следует ли выделять герпетиформную пузырчатку?

В некоторых классификациях герпетиформную пузырчатку рассматривают как вариант вульгарной или листовидной пузырчатки, однако клиническая картина слишком отличается от них, поэтому целесообразно рассматривать ее как самостоятельную фрорму.

7. Оправдано ли подразделять вульгарную пузырчатку на слизистый (изолированное поражение слизистой) и слизисто-кожный типы в связи с тем, что при слизистом типе выявляются аутоантитела к Dsg-1, а при слизисто-кожном - аутоантитела к Dsg-1, Dsg-3?

Мы считаем это нецелесообразным по следующим причинам: во-первых, исследования 2019 г. показали, что при изолированном поражении слизистой оболочки полости рта при вульгарной пузырчатке могут выявляться аутоантитела к Dsg-3 [17], во-вторых, в подавляющем большинстве случаев развиваются поражения кожи на фоне вовлечения слизистых оболочек, в-третьих, лечение этих фрорм практически не отличается.

8. Добавить ли в классификацию раздел «Другие

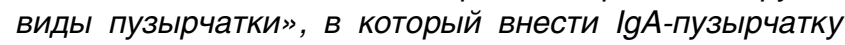
в силу того, что патогенез этой фрормы кардинально отличается от истинной пузырчатки?

Принято решение не усложнять классификацию, выделяя другие виды пузырчатки, поскольку в самом термине заложено отличие IgA-пузырчатки от истинной, кроме того, такой подход мы не встретили ни в одной классификации.

Обсуждение основных отечественных и зарубежных классификаций пузырчатки показало, что в их основе лежит выделение нозологических форм на основании клинико-морфологических критериев, данных прямой РИФ и реакции иммунопреципитации. Различия классификаций касаются в основном отдельных фрорм (лекарственно-индуцированная пузырчатка), подтипов (вегетирующая пузырчатка Ноймана и Аллопо; разделение IgA-пузырчатки на субкорнеальный и интрадермальный типы) или введения дополнительного раздела (другие виды пузырчатки), что в ряде случаев приводило, по мнению членов комиссии, к усложнению классификаций.

\section{Заключение}

В результате обсуждения различных вариантов классификаций пузырчатки рабочая группа Комитета РОДВК по классификациям в дерматовенерологии предлагает использовать в клинической работе врачей-дерматовенерологов; в учебных и научных целях - при обучении в медицинских образовательных учреждениях высшего образования, при обучении по специальности «Дерматовенерология» (код ОКСО 3.31.08.32), при планировании и проведении научных исследований, при публикациях в научных медицинских журналах, при разработке Клинических рекомендаций следующую классификацию пузырчатки:

\section{1. Вульгарная пузырчатка \\ 1.1. Вегетирующая пузырчатка \\ 2. Листовидная пузырчатка}

2.1. Эндемичная пузырчатка (Fogo selvagem)

2.2. Эритематозная пузырчатка (Senear - Usher)

3. Герпетиформная пузырчатка

4. Паранеопластическая пузырчатка

5. IgА-пузырчатка

5.1. Субкорнеальный пустулезный дерматоз

5.2. Внутриэпидермальный нейтрофильный дерматоз

\section{Литература/References}

1. Joly $P$, Horvath $B$, Patsatsi $A$, et al. Updated S2K guidelines on the management of pemphigus vulgaris and foliaceus initiated by the european academy of dermatology and venereology (EADV). J Eur Acad Dermatol Venereol. 2020;34(9):1900-1913. doi:10.1111/jdv.16752
2. Porro AM, Seque CA, Ferreira MCC, Enokihara MMSES. Pemphigus vulgaris. An Bras Dermatol. 2019;94(3):264-278.

doi:10.1590/abd1806-4841.20199011 
3. Egami S, Yamagami J, Amagai M. Autoimmune bullous skin diseases, pemphigus and pemphigoid. J Allergy Clin Immunol. 2020;145(4):1031-1047. doi.org/10.1016/j.jaci.2020.02.013

4. Lever WF. Pemphigus. Medicine (Baltimore). 1953;32(1):1-123. doi:10.1097/00005792-195302000-00001

5. Jablonska S, Chorzelski TP, Beutner EH, Chorzelska J. Herpetiform pemphigus, a variable pattern of pemphigus. Int J Dermatol. 1975;14(5):353-359. doi:10.1111/j.1365-4362.1975.tb00125.x

6. Wallach D, Foldès C, Cottenot F. Pustulose sous-cornée, acantholyse superficielle et IgA monoclonale [Subcorneal pustulosis, superficial acantholysis and monoclonal IgA]. Ann Dermatol Venereol. 1982;109(11):959-963 (In French).

7. Robinson ND, Hashimoto $T$, Amagai M, Chan LS. The new pemphigus variants. J Am Acad Dermatol. 1999;40(5 Pt 1):649-673.

doi:10.1016/s0190-9622(99)70145-3

8. Anhalt GJ, Kim SC, Stanley JR, et al. Paraneoplastic pemphigus. An autoimmune mucocutaneous disease associated with neoplasia. N Engl J Med. 1990;323(25):1729-1735.

doi:10.1056/NEJM199012203232503

9. Anhalt GJ. Paraneoplastic pemphigus. Adv Dermatol. 1997;12:77-97.

10. Wojnarowska $F$, Venning VA. Immunobullous Diseases. In: Burns T, Breathnach S, Cox N, Griffiths C 8th, editors. Rook's Textbook of Dermatology. Blackwell Publishing Ltd.; 2010.
11. Самцов А.В., Белоусова И.Э. Буллезные дерматозы. СПб.: 000 «Издательско-полиграфическая компания «КОСТА»; 2012. [Samtsov AV, Belousova IE. Bulleznye dermatozy. Saint Petersburg: KOSTA; 2012 (In Russ.)]

12. Hertl M, Jedlickova H, Karpati S, et al. Pemphigus. S2 Guideline for diagnosis and treatment - guided by the European Dermatology Forum (EDF) in cooperation with the European Academy of Dermatology and Venereology (EADV). J Eur Acad Dermatol Venereol. 2015;29(3):405-414. doi:10.1111/jdv.12772

13. Marcel F. Jonkman. Autoimmune Bullous Diseases. Text and Review. Switzerland: Springer International Publishing; 2016.

14. Махнева Н.В., Теплюк Н.П., Белецкая Л.В. Аутоиммунная пузырчатка. От истоков развития до наших дней. Екатеринбург: Издательские решения; 2016. [Mahneva NV, Tepljuk NP, Beleckaja LV. Autoimmunnaja puzyrchatka. Ot istokov razvitija do nashih dnej. Ekaterinburg: Izdatel'skie reshenija; 2016 (In Russ).]

15. Korman N. Pemphigus. J Am Acad Dermatol. 1988;18(6):12191238. doi:10.1016/s0190-9622(88)70128-0

16. Megahed M. Histopathology of Blistering Diseases: Springer; 2004;174-122. doi:10.1007/978-3-642-18651-6

17. Endo $H$, Rees TD, Niwa $H$, et al. Acantholysis may precede elevation of circulating anti-desmoglein 3 antibody levels in pemphigus vulgaris presenting with desquamative gingivitis. Clin Exp Dent Res. 2019;5(3):219-224. doi:10.1002/cre2.174

Участие авторов: концепция и дизайн статьи, поисково-аналитическая работа, написание статьи, прочтение, одобрение рукописи и направление рукописи на публикацию - A.В. Самцов; написание статьи, прочтение, одобрение рукописи и направление рукописи на публикацию Е.В. Соколовский; анализ литературных данных, прочтение, одобрение рукописи и направление рукописи на публикацию - Н.П. Теплюк; анализ литературных данных, прочтение, одобрение рукописи и направление рукописи на публикацию - И.Э. Белоусова; анализ литературных данных, прочтение, одобрение рукописи и направление рукописи на публикацию - М.М. Кохан; анализ литературных данных, прочтение, одобрение рукописи и направление рукописи на публикацию - Е.В. Матушевская.

Authors' participation: the concept and design of the article, search and analytical work, writing the article, reading, approving the manuscript and sending the manuscript for publication - Alexey V. Samtsov; writing the article, reading, approving the manuscript and sending the manuscript for publication - Evgeny V. Sokolovskiy; analysis of literary data, reading, approving the manuscript and sending the manuscript for publication — Natalia P. Teplyuk; analysis of literary data, reading, approving the manuscript and sending the manuscript for publication — Irena E. Belousova; analysis of literary data, reading, approving the manuscript and sending the manuscript for publication - Muza M. Kokhan; analysis of literary data, reading, approving the manuscript and sending the manuscript for publication — Elena V. Matushevskaya.

\section{Информация об авторах}

*Алексей Викторович Самцов - д.м.н., профессор; адрес: Россия, 194044, г. Санкт-Петербург, ул. Академика Лебедева, д. 2; ORCID iD: https://orcid.org/0000-0002-9458-087; eLibrary SPIN: 2287-5062; e-mail: avsamtsov@mail.ru

Евгений Владиславович Соколовский - д.м.H., професcop; ORCID iD: https://orcid.org/0000-0001-7610-6061; eLibrary SPIN: 6807-7137; e-mail: s40@mail.ru

Наталия Павловна Теплюк - д.м.н., профеccop; ORCID iD: https://orcid.org/0000-0002-5800-4800; eLibrary SPIN: 8013-3256; e-mail: teplyukn@gmail.com

Ирена Эдуардовна Белоусова - д.м.н., профресcop; ORCID iD: https://orcid.org/0000-0002-4374-4435; eLibrary SPIN: 6386-1117; e-mail: irena.belousova@mail.ru

Муза Михайловна Кохан - д.м.H., профресcop; ORCID iD: https://orcid.org/0000-0001-6353-6644; eLibrary SPIN: 3470-9306; e-mail: mkokhan@yandex

Елена Владиславовна Матушевская - д.м.H., професcop; ORCID iD: https://orcid.org/0000-0003-4583-0617; eLibrary SPIN: 7430-2112; e-mail: matushevskaya@mail.ru 


\section{Information about the authors}

*Alexey V. Samtsov - MD, Dr. Sci. (Med.), Professor; address: 2 Akademika Lebedeva street, 194044, Saint Petersburg, Russia; ORCID iD: https://orcid.org/0000-0002-9458-087; eLibrary SPIN: 2287-5062; e-mail: avsamtsov@mail.ru

Evgeny V. Sokolovskiy - MD, Dr. Sci. (Med.), Professor; ORCID iD: https://orcid.org/0000-0001-7610-6061; eLibrary SPIN: 6807-7137; e-mail: s40@mail.ru

Natalia P. Teplyuk - MD, Dr. Sci. (Med.), Professor; ORCID iD: https://orcid.org/0000-0002-5800-4800; eLibrary SPIN: 8013-3256; e-mail: teplyukn@gmail.com

Irena E. Belousova - MD, Dr. Sci. (Med.), Professor; ORCID iD: https://orcid.org/0000-0002-4374-4435; eLibrary SPIN: 6386-1117; e-mail: irena.belousova@mail.ru

Muza M. Kokhan - MD, Dr. Sci. (Med.), Professor; ORCID iD: https://orcid.org/0000-0001-6353-6644; eLibrary SPIN: 3470-9306; e-mail: mkokhan@yandex

Elena V. Matushevskaya - MD, Dr. Sci. (Med.), Professor; ORCID iD: https://orcid.org/0000-0003-4583-0617; eLibrary SPIN: 7430-2112; e-mail: matushevskaya@mail.ru 